Pacific Journal of Mathematic 


\title{
PERTURBATION OF DIFFERENTIAL OPERATORS
}

\author{
HenRy P. KRAMER
}

Introduction. N. Dunford, in a series of papers $[3,4,5]$, has initiated the study of operators on Banach spaces that allow a representation analogous to the Jordan canonical form for operators on a finite dimensional vector space. Such operators he has called spectral operators. They include, of course, self-adjoint operators which have found such wide application to problems of analysis. J. Schwartz [9] has exhibited an interesting class of spectral operators which contains many classical ordinary differential operators. His chief tool was a perturbation theorem that guarantees that if $T$ is a regular spectral operator with a discrete spectrum that converges to infinity sufficiently rapidly and $B$ is a bounded operator, then $T+B$ is again a regular spectral operator. This result provides a tool for showing that second order differential operators with suitable boundary conditions are regular spectral but does not suffice for proving this property for differential operators of higher order. This paper refines the method of J. Schwartz to allow application also to differential operators of higher order by showing that under certain conditions a regular spectral operator $T$ may be perturbed by an unbounded operator $S$ with the result that $T+S$ is still regular spectral.

The paper is divided into three parts. The first part presents preliminary notions and lemmas to be used in part II where the principal theoretical tool is fashioned in Theorem 1. Its object is to set forth conditions under which an operators is spectral (see Definition 1). This problem is attacked in the following form. Suppose that $T$ is known to be a spectral operator. Under what hypotheses on $T$ and a perturbing operator $S$ may it be said that the operator $T+S$ is spectral? An answer to this question is given in Theorem 1. This theorem is then applied in the third part to differential operators of even order with "separated" boundary conditions on a finite interval. First, the simple operator defined by means of the formal differential operator $d d^{2 \mu}$ and "separated" boundary conditions is shown to be spectral. Then, with the aid of Theorem 1, the perturbed operator

$$
d x^{2 \mu}+\sum_{i=2}^{2 \mu} Q_{i} \frac{d^{2 \mu-i}}{d x^{2 \mu-i}}
$$

Received August 21, 1956. This paper constitutes part of the author's doctoral dissertation submitted to the University of California, Berkeley, 1954, prepared under the guidance of Professor František Wolf 
where $Q_{i}$ may be any bounded operator on $\mathscr{C}_{2}(0,1)$ is seen to be spectral as well.

1. Preliminaries. N. Dunford [3, p. 560] has laid down the following.

Definition 1. Let $X$ be Banach space and $T$ a transformation on $X$ to $X$. If $E(e)$ is an operator valued function of Borel sets in the complex plane and

(a) $E(e) E(g)=E(e \cap g), E\left(e^{\prime}\right)=I-E(e), T E(e)=E(e) T$,

(b) $E(e) x$ is completely additive in $e$ for each $x \in X$,

(c) the spectrum of $T$, with domain and range restricted to $E(e) X$, is contained in the closure of $e$, and

(d) there exists a constant $M$ such that for every Borel set $e\|E(e)\|$ $<M$, then $E(e)$ is called a resolution of the identity for $T$ and $T$ is called a spectral operator.

The preceding definition covers a wide class of operators. In what is to follow, attention is focussed on a very restricted subset consisting of the regular spectral operators. The meaning of the adjective regular is clarified as follows.

Definition 2. An operator $T$ is regular if the resolvent set $\rho(T)$ $\neq \phi$ and if for some $\lambda \in \rho(T),(T-\lambda)^{-1}$ is completely continuous. (To be abbreviated c.c.)

Note that the spectrum of the c.c. operator $R_{\lambda}(T) \equiv(T-\lambda)^{-1}$ consists of a sequence of isolated points converging to 0 .

It follows by the spectral mapping theorem [12, p. 324 et seq.] that the spectrum of $T$ consists of a sequence of points $\lambda_{n}$ converging to $\infty$.

In the sequel, the condition

$$
I=\sum_{h=1}^{\infty} E\left(\lambda_{h}\right)
$$

shall sometimes be made in regard to the spectral measure of a regular spectral operator $T$. The above condition asserts that the spectral measure corresponding to the point at infinity is the null operator or $\mu=0$ is not an eigenvalue of $T^{-1}$. The existence of $T^{-1}$ as a c.c. operator may be assumed without loss of generality in view of the following.

Lemma 1. If $\lambda_{11} \in \rho(T)$ and $R_{\lambda_{0}}(T)$ is c.c., then $R_{\lambda}(T)$ is c.c. for all $\lambda \in \rho(T)$.

Proof. The first resolvent identity [6, p. 99] states that for $\lambda_{0} \in \rho(T)$ 
and $\lambda \in \rho(T)$

$$
R_{\lambda}(T)=R_{\lambda_{0}}(T)+\left(\lambda-\lambda_{0}\right) R_{\lambda}(T) R_{\lambda_{0}}(T)
$$

The product of a bounded operator and a c.c. operator is c.c. and the sum of two c.c. operators is again c.c. Thus it is apparent from (1) that $R_{\lambda}(T)$ is c.c. for all $\lambda \in \rho(T)$.

LEMMA 2 . If $S$ is a closed operator and $B$ is a bounded operator and $\mathscr{O}(S) \supset \mathscr{R}(B)$, then $S B$ is bounded.

Proof. $S B$ is closed. For suppose that $x_{n} \rightarrow x$ and $S B x_{n} \rightarrow y$. Since $B$ is continuous, $B x_{n} \rightarrow B x$. But since $S$ is closed $S\left(B x_{n}\right) \rightarrow S(B x)=(S B) x$ $=y$. Thus $S B$ is a closed operator defined on all of $X$ and therefore by virtue of the closed graph theorem $[1, p .41]$ it is bounded.

LEMмA 3. Let $J$ be a finite set of integers and suppose that $B_{n}$ is a set of bounded operators and $E_{n}$ a set of mutually orthogonal projections", both sets being indexed by J. Then

$$
\left\|\sum_{n \in J} E_{n} B_{n}\right\|^{2} \leq \sum_{n \in J}\left\|B_{n}\right\|^{2}
$$

Proof. Let $f \in H$ and $\|f\|=1$. It is an easy consequence of the Hermitian nature of $E_{n}$ and Schwarz' Lemma that

$$
\begin{aligned}
& \left\|\sum_{n \in . I} E_{n} B_{n} f\right\|^{2}=\sum_{n \in J} \sum_{k \in J}\left(E_{n} B_{n} f, E_{k} B_{k} f\right) \\
& \leq \sum_{n \in J}\left|\left(B_{n} f, E_{n} B_{n} f\right)\right| \leqq \sum_{n \in J}\left\|B_{n} f\right\|\left\|E_{n} B_{n} f\right\| \\
& \leq \sum_{n \in J}\left\|B_{n}\right\|^{2} .
\end{aligned}
$$

In the sequel, reference shall be made several times to the following.

Condition A. All but a finite number of the idempotents ${ }^{2} E\left(\lambda_{k}\right)$ associated with the points of the spectrum of $T$ project onto a onedimensional range and

$$
I=\sum_{k=1}^{\infty} E\left(\lambda_{h}\right)
$$

For a regular spectral operator, the last statement is equivalent to the assertion that the range of

${ }^{1}$ If $T$ is an operator then $\mathscr{P}(T)$ denotes its domain and $\mathscr{R}(T)$ its range.

2 An idempotent is an operator $E$ such that $E=E^{2}$. Idempotents $E_{1}$ and $E_{2}$ will be called orthogonal if $E_{1} E_{2}=0$. If $E_{1}=E^{*}$, then $E$ is a projection. 


$$
E_{\infty}=I-\sum_{1}^{\infty} E\left(\lambda_{k}\right)
$$

consists only of the null vector.

Condition B. Let $d_{k}$ denote the distance between $\lambda_{k}$ and the rest of the spectrum of $T$. Then there exists a number $\tau>0$ such that

$$
\sum_{k=1}^{\infty} d_{k}^{-\tau}<\infty .
$$

For use in the theorem to follow, it is necessary to define explicitly the concept of a fractional power for the special class of operators with which the theorem is concerned.

In this definition an application shall be made of a theorem of Lorch [8] and Mackey which asserts that if $E(e)$ is a uniformly bounded spectral measure, then there exists a nonsingular transformation of Hilbert space into itself such that $W E(e) W^{-1}$ is a Hermitian spectral measure.

Let $T$ be a regular spectral operator on Hilbert space $H$ which satisfies Condition A. Let $\mathscr{P}$ be the finite set of characteristic values $\lambda$ for which the idempotents $E(\lambda)$ project onto ranges of multiple dimension. Let $W$ be the automorphism of $H$ into itself which carries the spectral measure $E(e)$ of $T$ into the Hermitian spectral measure $E^{\prime}(e)$ $=W E(e) W^{-1}$ of $T^{\prime}=W T W^{-1}$.

Since $E^{\prime}(\mathscr{P}) \perp I-E^{\prime}(\mathscr{P})$, the two projections effect a unique decomposition of $H$ into a direct sum

$$
H=H_{1} \oplus H_{2}
$$

where

$$
H_{1}=E^{\prime}(\mathscr{P}) H
$$

and

$$
H_{2}=\left\{I-E^{\prime}(\mathscr{P})\right\} H .
$$

Now

$$
T^{\prime}=T_{1}^{\prime}+T_{2}^{\prime}
$$

where

$$
T_{1}^{\prime}=T^{\prime} E^{\prime}(\mathscr{P})
$$

and

$$
T_{2}^{\prime}=T^{\prime}\left\{I-E^{\prime}(\mathscr{P})\right\} .
$$


Upon restricting the domain of $T_{1}^{\prime}$ to $H_{1}$ and that of $T_{2}^{\prime}$ to $H_{2}$ one is confronted by a finite dimensional operator $\hat{T}_{1}^{\prime}$ and a normal operator $\hat{T}_{2}^{\prime}$.

If $-1<\nu<1$, the function $f(\lambda)=\lambda^{\nu}$ of the complex variable $\lambda$ is regular on the spectrum of $\hat{T}_{1}^{\prime}$ provided $0 \notin \sigma(T)$ (which is no essential limitation of generality) and $f(\lambda)$ is restricted to its principal value.

Then, following Dunford [4], one defines

$$
\left(\hat{T}_{1}^{\prime}\right)^{\nu}=\sum_{i=1}^{P} \sum_{m=0}^{\mu_{i}-1} \frac{\left(\hat{T}_{1}^{\prime}-\lambda_{i}\right)^{m}}{m !}\left\{\nu(\nu-1) \cdots(\nu-m+1) \lambda_{i}^{\nu-m} \hat{E}^{\prime}\left(\lambda_{i}\right)\right.
$$

where $\mu_{i}$ is the order of the pole $\lambda_{i}$ or the resolvent and $\hat{E}^{\prime}\left(\lambda_{i}\right)$ is the restriction of $E^{\prime}\left(\lambda_{i}\right)$ to $E^{\prime}(\mathscr{T}) H$. Since $T_{2}^{\prime}$ is normal one has the spectral decomposition

$$
\hat{T}_{2}^{\prime}=\sum_{k=1}^{\infty} \lambda_{h} \hat{E}^{\prime}\left(\lambda_{k}\right)
$$

and by the operational calculus for normal operators (cf. [10, pp. 4851] for example)

$$
\left(\hat{T}_{2}^{\prime}\right)^{\nu}=\sum_{k=1}^{\infty} \lambda_{k}^{\nu} \hat{E}^{\prime}\left(\lambda_{k}\right)
$$

Now define $\left(T_{1}^{\prime}\right)^{\nu}$ and $\left(T_{2}^{\prime}\right)^{\nu}$ by the rules

$$
\begin{aligned}
& f_{1} \in H_{1} \Rightarrow\left(T_{1}^{\prime}\right)^{\nu} f_{1}=\left(\hat{T}_{1}^{\prime}\right)^{\nu} f_{1} \quad\left(T_{2}^{\prime}\right)^{\nu} f_{1}=0 \\
& f_{2} \in H_{2} \Rightarrow\left(T_{1}^{\prime}\right)^{\nu} f_{2}=0 \quad\left(T_{2}^{\prime}\right)^{\nu} f_{2}=\left(\hat{T}_{2}^{\prime}\right)^{\nu} f_{2} .
\end{aligned}
$$

Then

$$
\left(T^{\prime}\right)^{\nu} \equiv\left(T_{1}^{\prime}\right)^{\nu}+\left(T_{2}^{\prime}\right)^{\nu}
$$

and finally,

$$
T \equiv W^{-1}\left(T^{\prime}\right)^{\nu} W
$$

The proof of the perturbation theorem below strongly depends on the operational calculus for spectral operators developed by N. Dunford and explicitly adapted to the case at hand by J. Schwartz [9]. For the sake of ready reference the pertinent results are presented here.

If $T$ is a regular operator with a finite set of characteristic numbers

$$
\left\{\lambda_{1}, \lambda_{2}, \cdots, \lambda_{n}\right\}
$$

which are multiple poles of the resolvent and 


$$
\sum_{k=1}^{\infty} E\left(\lambda_{k}\right)=I
$$

and $f(\lambda)$ is a complex-valued function which is uniformly bounded on the spectrum of $T$ and possesses the required derivatives, then

$$
f(T) \equiv \sum_{i=1}^{n} \sum_{j=0}^{\nu_{i}-1} \frac{f^{(j)}\left(\lambda_{i}\right)}{j !}\left(T-\lambda_{i}\right)^{j} E\left(\lambda_{i}\right)+\sum_{i=n+1}^{\infty} f\left(\lambda_{i}\right) E\left(\lambda_{i}\right) .
$$

For such an $f(T)$ Dunford [5] has shown that the series defining it converges in the strong operator topology and that there exists a constant $K(T)$ such that

$$
\|f(T)\| \leq K \cdot \max _{\lambda \in \sigma(T)}|f(\lambda)| .
$$

On the basis of this result J. Schwartz [9] enunciates the following.

LEMMA. If $S$ is a regular spectral operator all but a finite set of whose eigenvalues $\lambda_{n}$ are simple poles of the resolvent, and if $S$ also satisfies

$$
\sum_{i=1}^{\infty} E\left(\lambda_{i}\right)=I
$$

then there exists an absolute constant $K$ such that

$$
\left\|(\lambda-S)^{-1}\right\| \leq K / \operatorname{dist}(\lambda, \sigma(S))
$$

for all $\lambda$ not within a fixed radius $\varepsilon$ of any multiple pole of the resolvent.

In the theorem below let it be understood that

$$
\mathscr{X}(T+S) \equiv \mathscr{X}(T) \cap \mathscr{X}(S) \text {. }
$$

2. The perturbation theorem. The principal result of the present paper is the following.

THEOREM 1. Let $T$ be a regular spectral operator on Hilbert space. $H$ and suppose that it satisfies conditions $A$ and $B$. Let $S$ be such $a$, closed operator that for some $\nu, 0<\nu<1, \mathscr{X}(S) \supset \mathscr{D}\left(T^{\nu}\right)$ and $\mathscr{D}\left(S^{*}\right) \supset$ Ø $\left(T^{* \nu}\right)$. Moreover, suppose that for all but a finite set $P$ of positive integers, for all

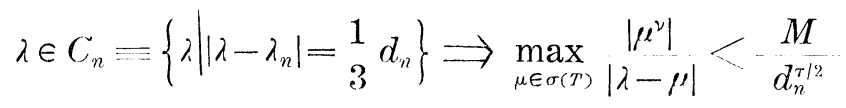

Under the above hypotheses, $T+S$ is again a regular spectral operator. 
Proof: Since, for $\lambda \in \rho(T)$,

$$
\mathscr{R}\left(R_{\lambda}(T)\right)=\mathscr{Z}(T) \subset \mathscr{Q}\left(T^{\nu}\right) \subseteq \mathscr{V}(S),
$$

$S R_{\lambda}(T)$ is well defined and is, in fact, by Lemma 2 , a bounded operator. By the same token $S T^{-\nu}$ is bounded. In order to show that $T+S$ is regular, it need merely be ascertained that $R_{\lambda}(T+S)$ is c.c. at one point $\lambda \in \rho(T)$ and for this purpose we examine the formula

$$
R_{\lambda}(T+S)=R_{\lambda}(T)\left\{I-S R_{\lambda}(T)\right\}^{-1}
$$

which is valid for $\lambda \in \rho(T)$ provided only that $\left\{I-S R_{\lambda}(T)\right\}^{-1}$ exists. If, $\left\{I-S R_{\lambda}(T)\right\}^{-1}$ not only exists but is also bounded, then $R_{\lambda}(T+S)$ as the product of a c.c. and a bounded operator is itself c.c.

But the hypotheses of the theorem allow one to state that $\left\{I-S R_{\lambda}(T)\right\}^{-1}<2$ for $\lambda \in C_{n}$ and all $n$ sufficiently large. This is proved as follows

$$
S R_{\lambda}\left(T^{\prime}\right)=S T^{-\nu} T^{\nu}\{\lambda I-T\}^{-1}=S T^{-\nu}\left\{(\lambda I-T) T^{1-\nu}\right\}^{-1} .
$$

By Dunford's operational calculus and the hypotheses of the theorem it is true that

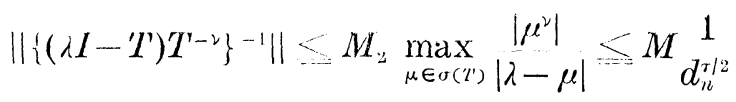

Let $\left\|S T^{-\nu}\right\|=M_{1}$. Then

$$
\left\|S R_{\lambda}\left(T^{\prime}\right)\right\| \leq \underset{d_{n}^{2 / 2}}{M_{1} M_{2}}=\frac{M}{d_{n}^{7 / 2}}
$$

and since in view of Condition $\mathrm{B}, \lim d_{n}^{-\tau / 2}=0$, one has for all $n$ sufficiently large $\left\|S R_{\lambda}(T)\right\|<1 / 2$ while $\lambda \in C_{n}$. From this estimate follows the possibility of expanding

$$
\left\{I-S R_{\lambda}(T)\right\}^{-1}=I+S R_{\lambda}(T)+\left(S h_{\lambda}^{\prime}(T)\right)^{2}+\cdots
$$

in an absolutely convergent series so that

$$
\left\|\left\{I-S R_{\lambda}(T)\right\}^{-1}\right\|<\frac{1}{1-1 / 2}=2 .
$$

Il is immediate from the above that if $\lambda$ lies outside the assemblage of circles $C_{k}$, then for each $\mu_{k} \in \sigma\left(T^{\prime}\right)$ we have

$$
\frac{\left|\mu_{k}^{2}\right|}{\left|\lambda-\mu_{k}\right|}<\frac{\left|\mu_{k}^{2}\right|}{\left|\lambda^{\prime}-\mu_{k}\right|}
$$

where $\lambda^{\prime}$ is the intersection of the line connecting $\lambda$ with $\mu_{k}$ and the 
circle $C_{k}$. From this, the above estimates follow a fortiori. Consequently, except for a finite set, all points of $\sigma(T+S)$ lie inside the circles $C_{k}$.

In order to show that the spectral measure $\left\{E^{\prime}\left(\lambda_{k}^{\prime}\right)\right\}$ of $T+S$ is uniformly bounded it is convenient to assume that the spectral measure $\left\{E\left(\lambda_{k}\right)\right\}$ of $T$ consists of Hermitian idempotents, that is, that $E\left(\lambda_{k}\right)=E\left(\lambda_{k}\right)^{*}$. That this assumption may be made without sacrificing generality is due to the theorem of Lorch-Mackey. It must be verified that if $T$ and $S$ satisfy the conditions of the theorem so do $T^{\prime}=W T W^{-1}$ and $S^{\prime}=W S W^{-1}$.

(a) $\sigma\left(T^{\prime}\right)=\sigma(T)$. For suppose $\lambda \in \rho(T)$. Then $R_{\lambda}(T)$ is a bounded operator. But

$$
W(\lambda I-T)^{-1} W^{-1}=\left(\lambda I-W T W^{-1}\right)^{-1} \equiv\left(\lambda I-T^{\prime}\right)
$$

is also bounded. Hence $\rho(T)=\rho\left(T^{\prime}\right)$ and the result follows on taking complements with respect to the extended complex plane.

$$
\operatorname{dim} W E\left(\lambda_{k}\right) W^{-1}=\operatorname{dim} W E\left(\lambda_{k}\right) \leqq \operatorname{dim} E\left(\lambda_{k}\right) .
$$

However, since $W$ is continuous, with a continuous inverse, it maps no nonzero vector into zero and thus, since $\operatorname{dim} E\left(\lambda_{k}\right)=1$ for almost all $k$, the same is true with regard to $W E\left(\lambda_{k}\right) W^{-1}$. Also

$$
0=W\left(I-\sum_{k=1}^{\infty} E\left(\lambda_{k}\right)\right) W^{-1}=I-\sum_{k=1}^{\infty} W E_{k} W^{-1}
$$

(c) $f \in \mathscr{D}\left(W T^{\nu} W^{-1}\right) \Rightarrow W^{-1} f^{\prime} \in \mathscr{D}\left(T^{\nu}\right) \Rightarrow W^{-1} f \in \mathscr{D}(S) \Rightarrow f^{\prime} \in \mathscr{O}\left(S^{\prime}\right)$

and similarly for the adjoints.

In the remainder of the proof it shall, therefore, be supposed that the spectral family $E\left(\lambda_{k}\right)$ consists of Hermitian idempotents. For convenience, the primes introduced above shall be suppressed.

The proof of uniform boundedness rests on the formula

$$
\begin{aligned}
& R_{\lambda}(T+S)-R_{\lambda}(T) \\
& \quad=R_{\lambda}(T) S R_{\lambda}(T)+R_{\lambda}(T) S R_{\lambda}(T) S R_{\lambda}(T)\left\{I-S R_{\lambda}(T)\right\}^{-1}
\end{aligned}
$$

which is easily obtained from (1) and the operator analogue of $1 /(1-x)$ $=1+x+x^{2} /(1-x)$, and on the basic relation

$$
E\left(\lambda_{n}\right)=\frac{1}{2 \pi i} \oint_{C_{n}} R_{\lambda}(T) d \lambda .
$$

Let $J$ be a finite set of positive integers all of which are sufficiently large that is, $N \in J \Rightarrow N>N_{0}$. The nature of $N_{0}(T, S)$ will be specified somewhat more precisely in the sequel. Then, on integrating both members of (4) one finds that 
(6)

$$
\begin{aligned}
& \left\|\sum_{n \in J} E_{n}^{\prime}-E\left(\lambda_{n}\right)\right\| \leqq\left\|\sum_{n \in J} \frac{1}{2 \pi i} \oint_{C_{n}} R_{\lambda}(T) S R_{\lambda}(T) d \lambda\right\| \\
& \quad+\left\|\sum_{n \in J} \frac{1}{2 \pi i} \oint_{C_{n}} R_{\lambda}(T) S R_{\lambda}(T) S R_{\lambda}(T)\left\{I-S R_{\lambda}(T)\right\}^{-1} d \lambda\right\|
\end{aligned}
$$

where $E_{n}^{\prime}$ represents the spectral measure corresponding to that portion of the spectrum of $T+S$ which lies inside the circle $C_{n}$. In order to place bounds on the right member of (6) one employs a well established inequality for operator valued functions $A(\lambda)$ analytic on a contour $C$ of length $L[12$, p. 324].

$$
\left\|\oint_{C} A(\lambda) d \lambda\right\| \leqq \max _{\lambda \in C}\|A(\lambda)\| \cdot L .
$$

Applying this result to the second term of the right member of (6) one finds

$$
\begin{aligned}
\left\|\sum_{n \in J} \frac{1}{2 \pi i} \oint_{C_{n}} R_{\lambda}(T) S R_{\lambda}(T) S R_{\lambda}(T)\left\{I-S R_{\lambda}(T)\right\}^{-1} d \lambda\right\| \\
\leqq \sum_{n \in J} \frac{1}{2 \pi}\left\|R_{\lambda}(T)\right\|\left\|S R_{\lambda}(T)\right\|^{2}\left\|\left\{I--S R_{\lambda}(T)\right\}^{-1}\right\| \cdot \frac{1}{3} d_{n} .
\end{aligned}
$$

Now using inequalities (2) and (3) to estimate $\left\|\left\{I-S R_{\lambda}(T)\right\}^{-1}\right\|$ and $\left\|S R_{\lambda}(T)\right\|$ and Lemma 3 of J. Schwartz reproduced above, one obtains for this term the bound

$$
\frac{1}{2 \pi n \in J} \sum_{n=5} \frac{M^{2}}{d_{n}^{\tau}} \leqq \frac{M^{2}}{2 \pi} \sum_{n=1}^{\infty} \frac{1}{d_{n}^{\tau}}<\infty
$$

The term

$$
\left\|\sum_{n \in J} \frac{1}{2 \pi i} \oint_{C_{n}} R_{\lambda}(T) S R_{\lambda}(T) d \lambda\right\|
$$

requires closer investigation. By employing the representation

$$
R_{\lambda}(T)=\frac{E\left(\lambda_{n}\right)}{\lambda-\lambda_{n}}+R^{\jmath}\left(\lambda_{n}\right)+A_{n}\left(\lambda-\lambda_{n}\right)
$$

where $A_{n}\left(\lambda-\lambda_{n}\right)$ is a power series in $\lambda-\lambda_{n}$ without constant term and, applying the residue theorem, one finds that

$$
\frac{1}{2 \pi i} \oint_{C_{n}} R_{\lambda}(T) S R_{\lambda}(T) d \lambda=R^{\mathrm{j}}\left(\lambda_{n}\right) S E\left(\lambda_{n}\right)+E\left(\lambda_{n}\right) S R^{0}\left(\lambda_{n}\right) .
$$

It remains to find bounds for 


$$
\left\|\sum_{n \in J} E\left(\lambda_{n}\right) S R^{0}\left(\lambda_{n}\right)\right\|
$$

and

$$
\left\|\sum_{n \in J} R^{0}\left(\lambda_{n}\right) S E\left(\lambda_{n}\right)\right\|
$$

On observing that

$$
\left\|\sum_{n \in J} R^{\mathrm{v}}\left(\lambda_{n}\right) S E\left(\lambda_{n}\right)\right\|=\left\|\sum_{n \in J} E\left(\lambda_{n}\right) S^{*} R^{\mathrm{v}}\left(\lambda_{n}\right)^{*}\right\|
$$

and identifying $S R^{0}\left(\lambda_{n}\right)$ is one case and $S^{*} R^{0 *}\left(\lambda_{n}\right)$ in the other with $B_{n}$ of Lemma 3, one sees that the terms in question are bounded by

$$
\sum_{n \in J}\left\|S R^{0}\left(\lambda_{n}\right)\right\|^{2} .
$$

It is not difficult to estimate $\left\|S R^{0}\left(\lambda_{n}\right)\right\|$. Again turning to the device

$$
\left\|S R^{0}\left(\lambda_{n}\right)\right\| \leqslant\left\|S T^{-\nu}\right\|\left\|T^{\nu} R^{\prime \prime}\left(\lambda_{n}\right)\right\|
$$

and noting that

$$
R^{\prime \prime}\left(\lambda_{n}\right)=\lim _{\lambda \rightarrow \lambda_{n}} R_{\lambda}\left(T^{\prime}\right)-\frac{E\left(\lambda_{n}\right)}{\lambda-\lambda_{n}}
$$

one has

$$
R_{0}\left(\lambda_{n}\right)==\sum_{k \neq n} \frac{E\left(\lambda_{k}\right)}{\lambda_{n}-\lambda_{k}} .
$$

(In this formula, in order to avoid notational complications, the effect of the finite set of multiple poles of the resolvent has been neglected.) One sees that $R^{\prime \prime}\left(\lambda_{n}\right)=F^{\prime}(T)$, where $F^{\prime}(\lambda)$ is defined in the neighborhoods of the spectral points $\lambda_{k}$ as follows:

$$
F(\lambda)=\left\{\begin{array}{cl}
\frac{1}{\lambda_{n}-\lambda} & \lambda \text { near } \lambda_{k} k \neq n \\
0 & \lambda \text { near } \lambda_{n} .
\end{array}\right.
$$

Consequently, $T^{\prime 2} P^{\prime \prime}\left(\lambda_{n}\right)=G\left(T^{\prime}\right)$ with

$$
G(\lambda)=\left\{\begin{array}{cl}
\frac{\lambda^{2}}{\lambda_{n}-\lambda} & \lambda \text { near } \lambda_{k} k \neq n \\
0 & \lambda \text { near } \lambda_{n}
\end{array}\right.
$$

Now applying the bound arising from the operational calculus one obtains 


$$
\left\|S R^{\prime \prime}\left(\lambda_{n}\right)\right\| \leq\left\|S T^{-\nu}\right\|\left\|G\left(T^{\prime}\right)\right\| \leq M_{1} M_{2} \max _{\lambda \in \sigma\left(T^{\prime}\right)}|G(\lambda)|
$$

Let $\mu \in C_{n}$.

$$
\begin{aligned}
& \left|\begin{array}{c}
\lambda^{2} \\
\lambda_{n}-\lambda
\end{array}\right|<|| \lambda_{n}-\mu|-| \lambda-\mu \mid<\frac{\left|\lambda^{2}\right|}{|\lambda-\mu|\left|\frac{\left|\lambda_{n}-\mu\right|}{|\lambda-\mu|}-1\right|} \\
& \leq \frac{\left|\lambda^{2}\right|}{|\lambda-\mu|\left|\begin{array}{c}
\frac{1}{3} d_{n} \\
d_{n}
\end{array}\right|+\frac{3\left|\lambda^{2}\right|}{2|\lambda-\mu|}} .
\end{aligned}
$$

Using the hypothesis made with regard to this function one finally has

$$
\left\|S R^{0}\left(\lambda_{n}\right)\right\| \leq{ }_{2}^{3} M_{1} M_{2} \frac{1}{d_{n}^{\tau / 2}}=M^{\prime} \frac{1}{d_{n}^{\tau / 2}} .
$$

Now one is prepared to state that

$$
\left\|\sum_{n \in J} \frac{1}{2 \pi i} \oint_{C_{n}}^{\infty} R_{\lambda}(T) S R_{\lambda}(T) d \lambda\right\|^{*}<2 M^{2} \sum_{n=1}^{\infty} \frac{1}{d_{n}^{\tau}}<\infty .
$$

Thus

$$
\left\|\sum_{n \in J}\left(E_{n}^{\prime \prime}-E\left(\lambda_{n}\right)\right)\right\| \leq K
$$

If it were known that $E_{n}$ is the spectral measure corresponding to one point of the spectrum of $T+S$, the proof of uniform boundedness would be complete. The next few lines shall be devoted to showing that, indeed, except for a finite number of indices, in every circle of radius ${ }_{3}^{1} d_{n}$ about $\lambda_{n} \in \sigma(T)$ there lies exactly one point $\lambda_{n}^{\prime} \in o(T+S)$ and the spectral measure $E^{\prime}\left(\lambda_{n}^{\prime}\right)$ corresponding to this point has a one dimensional range.

In (6) let the index set $J$ have $n$ for its only member. Then one sees on examining the estimates of the bound of the right member of (6),

$$
\left\|E_{n-1}^{\prime-} \boldsymbol{E}\left(\lambda_{n}\right)\right\|<\begin{gathered}
K^{\prime} \\
d_{n}^{7 / 2}
\end{gathered}
$$

For $n$ sufficiently large

$$
\frac{K^{\prime}}{d_{n}^{\tau / 2}}<\frac{1}{2}
$$

which by Lemma 4 of [9] (also cf. [10, p. 320]) implies that $E_{n}^{\prime}$ and $E\left(\lambda_{n}\right)$ have the same dimension, which by hypothesis is 1 . Therefore, 
$(T+S) E_{n}^{\prime}$ considered as an operator on the range of $E_{n}$ is a scalar $\lambda_{n}^{\prime}$ and precisely one point $\lambda_{n}^{\prime} \in \sigma(T+S)$ lies in the circle $C_{n}$.

Thus $T+S$ is a regular operator with uniformly bounded spectral measure and is therefore a spectral operator. (cf. [9, Lemma 2].

From the foregoing proof flow two consequences deserving of explicit mention.

Conollary 1. The operator $T+S$ satisfies Condition $B$ and for all $n$ sufficiently large $\left|\lambda_{n}^{\prime}-\lambda_{n}\right|<\frac{1}{3} d_{n}$.

Proof. In virtue of the remark following inequality (3) of the proof, all but a finite number of the points of $\sigma(T+S)$ lie inside the circles $C_{n}$ with center at $\lambda_{n} \in \sigma(T)$ and radius $\frac{1}{3} d_{n}$. Moreover, the discussion following (3) shows that except for a finite number of indices exactly one point $\lambda_{n}^{\prime}$ of $\sigma(T+S)$ lies in the circle $C_{n}$ about $\lambda_{n}$. Now suppose $\lambda_{k}^{\prime} \in \sigma(T+S)$ and its nearest neighbor is $\lambda_{k-1}^{\prime} \in \sigma(T+S)$. Then

$$
\begin{aligned}
d_{k}^{\prime}=\left|\lambda_{k}^{\prime}-\lambda_{k-1}^{\prime}\right| & \leqq\left|\lambda_{k}^{\prime}-\lambda_{k}\right|+\left|\lambda_{k}-\lambda_{k-1}\right|+\left|\lambda_{k-1}-\lambda_{k-1}^{\prime}\right| \\
& \leqq 1 / 3 d_{k}+d_{k}+1 / 3 d_{k-1} \leqq 5 / 3 d_{k},
\end{aligned}
$$

and

$$
\sum_{m}^{\infty} d_{k}^{\prime-\tau} \leqq\left(\frac{3}{5}\right)^{\tau} \sum_{m}^{\infty} d_{k}^{-\tau}<\infty
$$

It is of importance to know whether the perturbed operator $T+S$ still enjoys the "completeness" property

$$
\sum_{k=1}^{\infty} E^{\prime}\left(\lambda_{k}^{\prime}\right) \doteq I
$$

with which the unperturbed operator $T$ is endowed by hypothesis. The answer is given in the following.

TheOREM 2. If $T$ and $S$ satisfy the conditions of Theorem 1, then

$$
\sum_{k=1}^{\infty} E^{\prime}\left(\lambda_{k}^{\prime}\right)=I
$$

Proof. The proof rests on Lemma 16 of [9] which states:

The space $S_{\infty}(T) \equiv\left\{f \mid\right.$ for each positive integer $\left.k, E\left(\lambda_{k}\right) f=0\right\}$ is the set of all $f \in H$ for which $f(\lambda)=R_{\lambda}(T) f$ is an entire function of $\lambda$.

Suppose $C$ is a contour in the complex plane whose minimum distance from the spectrum $\sigma(T)$ is $d(C)$. Consider the function 


$$
f(\lambda, \mu)=\frac{\mu^{\nu}}{\lambda-\mu}
$$

for $\lambda \in C$ and $\mu \in \sigma(T)$. Now let $\lambda^{\prime} \in C$ be such that $\operatorname{dist}\left(\lambda^{\prime}, \sigma(T)\right)=d(C)$. and let $\mu_{n}$ be the point in $\sigma(T)$ such that $\operatorname{dist}\left(\lambda^{\prime}, \mu_{n}\right)=d(C)$. Then

$$
|f(\lambda, \mu)| \leqq \frac{\left|\mu_{n}^{\nu}\right|}{\left|\lambda^{\prime}-\mu_{n}\right|}<1 / d_{n}^{\tau / 2} .
$$

By choosing $C$ properly one can achieve that $\left\|S R_{\lambda}(T)\right\|<1 / 2$ for $\lambda \in C_{n}$ and, therefore, a fortiori, for $\lambda \in C$. Hence, by (3) $\left\|\left\{I-S R_{\lambda}(T)\right\}^{-1}\right\|<2$ and by the above cited lemma, for $f \in S_{\infty}(T)$, one then has for $\lambda \in C$

$$
\begin{aligned}
\left\|R_{\lambda}(T+S) f\right\| & =\left\|R_{\lambda}(T)\left\{I-S R_{\lambda}(T)\right\}^{-1} f^{\prime}\right\| \\
& \leqq \frac{k^{\prime}}{d(C)}\|f\| .
\end{aligned}
$$

Now, given $\varepsilon>0$, choose $C$ in such a way that $k^{\prime} \mid d(C)<\varepsilon$. Then

$$
\left\|R_{\lambda}(T+S) f\right\|<\varepsilon\|f\| .
$$

The arbitrary nature of $\varepsilon$, the fact that $f(\lambda)=R_{\lambda}(T+S) f$ is an entire function of $\lambda$, and the permissible application of the maximum modulus principle allow one to assert that for all $\lambda$ in the interior of $C$,

$$
R_{\lambda}(T+S) f=0 \text {. }
$$

In particular at points $\lambda \in \rho(T), R_{\lambda}(T+S)$ has an inverse. There are such points in the interior of $C$. Thus $f=0$ and the theorem is proved.

3. Application to differential operators of even order. $N=2 \mu$. In appliying Theorems 1 and 2 to differential operators, the unperturbed operator $T$ is identified with the operator $\tau \equiv d^{N} / d x^{N}$ with domain restricted by the two considerations:

(a) $f \in \mathscr{D}(T)$ only if $f \in \mathrm{C}^{N-1}(0,1)$ and $\frac{d^{N-1} f}{d x^{N-1}}$ is absolutely continuous, and

(b) $f \in \mathscr{D}(T)$ only if $f$ satisfies $N=2 \mu$ linearly independent boundary conditions of which $\mu$ bear on the point $x=0$ and $\mu$ on the point $x=1$. These boundary conditions can always, by linear combinations, be brought to the form

$$
\begin{aligned}
A_{i}(f)=f^{\left(k_{i}\right)}(0)+\sum_{j=0}^{k_{i}-1} \alpha_{i j} f^{(1)}(0) \quad \begin{array}{r}
i \\
=1,2, \cdots, \mu \\
k_{1}>k_{2}>\cdots>k_{\mu}
\end{array}
\end{aligned}
$$




$$
B_{\imath}(f)=f^{\left({ }^{\prime}\left(i^{\prime}\right)\right.}(1)+\sum_{j=0}^{l_{i}-1} \beta_{i j} f^{(1)}(1)
$$

$$
\begin{array}{r}
i=1,2, \cdots, \mu \\
l_{1}>l_{2}>\cdots>l_{\mu}
\end{array}
$$

To show that $T$ is a regular operator it is most convenient to refer to Lemma 10 of $[9$, p. 434] which states:

Let $T$ be a differential operator and suppose that for some complex $\lambda$ both $T-\lambda$ and $T^{*}-\lambda$ have an inverse. Then $T$ and $T^{*}$ are regular operators, $T$ and $T^{*}$ have spectra related by $\sigma(T)=\sigma\left(T^{*}\right)$, and determine spectral measures $E_{1}$ and $E_{2}$ related by $E_{1}(\lambda)=E_{2}^{*}(\bar{\lambda})$.

Consider the differential equation $(\tau-\lambda) f=0$. By manipulating a tentative power series solution it can be shown in an elementary fashion that there exists a set of linearly independent solutions which are entire in the parameter $\lambda$. Let this set be $\left\{u_{1}, u_{2}, \cdots, u_{N}\right\}$. The general solution of the above equation can then be expressed in the form:

$$
f(x)=\sum_{i=1}^{N} C_{i} u_{i}(x, \lambda) .
$$

On imposing the $N$ linearly independent boundary conditions, one obtains a system of $N$ homogeneous equations in the $N$ unknowns $C_{i}$. This system has a nonvanishing solution vector if and only if the determinant of the matrix of the coefficients vanishes. This determinant, however, being a linear combination of entire functions in $\lambda$ is itself entire. Hence its zeroes are isolated. Thus, for all but a countable set of points $\lambda_{k}$, one finds that $f(x)=0$, and thus $(T-\lambda)^{-1}$ exists. But, since the adjoint operator also has exactly $N$ linearly independent boundary conditions associated with it, it follows by the same argument that there exists only a countable number of points $\mu_{k}$ where $\left(T^{*}-\mu_{n}\right)^{-1}$ fails to exist. Consequently, one can find a point $\lambda$ such that both $(T-\lambda)^{-1}$ and $\left(T^{*}-\bar{\lambda}\right)^{-1}$ exist and, therefore, by the cited lemma, $T$ is regular.

It shall now be verified that $T$ satisfies the spectral Condition $B$. This will be accomplished by showing that the above boundary conditions are what Birkhoff [2] has called regular. To clarify the meaning of this term the technique for obtaining an asymptotic development for the characteristic numbers and functions established in the general case by G. D. Birkhoff [2] and amplified and developed rigorously by J. Tamarkin shall here be briefly recapitulated.

Since there are $N$ linearly independent solutions of the equation

$$
\frac{d^{N} f}{d x^{N}}=\lambda f
$$

a solution of the boundary value problem must have the form 


$$
f(x)=\sum_{l=1}^{N} C_{l} u_{l}(x)
$$

The requirement that $A_{i}(f)=B_{i}(f)=0$ leads to a set of $N$ linear equations in $N$ unknowns $\left\{C_{l}\right\}$. A necessary and sufficient condition that a nontrivial solution $\left\{C_{l}\right\}$ of this system exist is the vanishing of the determinant of the coefficients:

$$
\Delta(\lambda)=\left|\begin{array}{ccc}
A_{1}\left(u_{1}\right) & \cdots & A_{1}\left(u_{N}\right) \\
\vdots & & \vdots \\
A_{\mu}\left(u_{1}\right) & \cdots & A_{\mu}\left(u_{N}\right) \\
B_{1}\left(u_{1}\right) & \cdots & B_{1}\left(u_{N}\right) \\
\vdots & & \vdots \\
B_{\mu}\left(u_{1}\right) & \cdots & B_{\mu}\left(u_{N}\right)
\end{array}\right| .
$$

It should be noted that the solution is unique provided not all of the first minors of $\Delta(\lambda)$ vanish, that is, in this case, the characteristic value is simple.

A fundamental set of solutions of the differential equation

$$
\begin{aligned}
& d^{N} f \\
& d x^{N}
\end{aligned}=\lambda f
$$

consists of

$$
u_{h}(x ; \lambda)=e^{\rho \omega_{k} x}
$$

where

$$
\lambda^{1 / N}=\rho=|\lambda|^{1 / N} e^{i(1 / N) \arg \lambda}
$$

and $\omega_{k}$ are the $N$ distinct $N$ th roots of unity. The transformation $\rho^{N}=\lambda$ transports the entire $\lambda$-plane into a sector of angular width $2 \pi / N$ of the $\rho$-plane. There is, then, a biunique correspondence between the zeroes of $\Delta(\lambda)$ in the $\lambda$-plane and the zeroes of $\delta(\rho) \equiv \Delta\left(\rho^{N}\right)$ in a sector of angular width $2 \pi / N$ in the $\rho$-plane.

The elements of the determinant $\delta(\rho)$ can, by (8) be written as follows:

$$
\begin{aligned}
& A,(u,)=\rho^{k}{ }_{i}\left\{\omega_{j}^{k_{i}}+\sum_{t=0}^{k_{,}-1} \begin{array}{l}
\alpha_{i t} \omega_{j}^{t} \\
\rho^{k_{i}}-t
\end{array}\right\}=\rho^{k},\left\{\omega_{j^{k}}^{k_{i}}+A_{i j}\right\} \\
& B_{i}\left(u_{,}\right)=\rho^{l}, e^{\rho \omega \omega_{i}}\left\{\omega_{j^{i}}^{l_{i}}+\sum_{s=0}^{l_{i}-1} \frac{\beta_{i s} \omega_{j}^{s}}{\rho^{l_{i}}-s}\right\}=\rho^{l} i e^{\rho \omega_{j}}\left\{\omega_{j^{i}}^{l_{i}}+B_{i,}\right\}
\end{aligned}
$$

where $\lim _{|\rho| \rightarrow \infty} A_{i, i}=\lim _{|\rho| \rightarrow \infty} B_{i, j}=0$. After removing the factors $\rho^{k_{i}}, \rho^{l_{i}}$ from 
the rows with index $i$ and $i+\mu$ one has

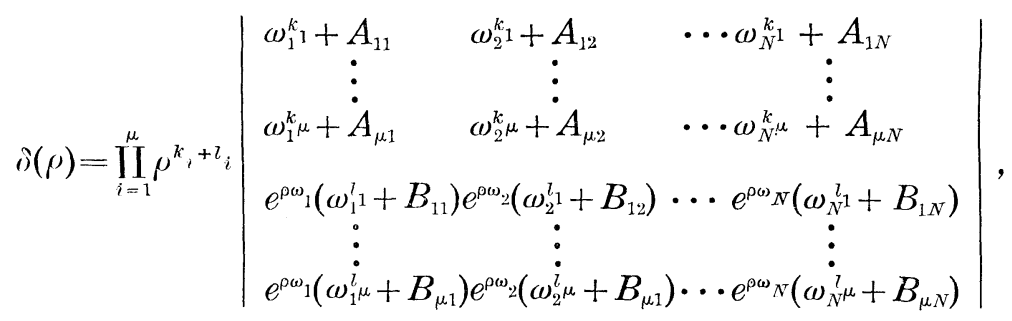

The sector $S$ shall now be chosen in a convenient fashion. To this end, it is proper to distinguish between two cases:

(1) $\mu$ is even

$$
S=\left\{\rho \mid-\frac{\pi}{2 \mu} \leq \arg \rho \leqq \frac{\pi}{2 \mu}\right\}
$$

(2) $\mu$ is odd

$$
S=\left\{\rho \mid 0 \leqq \arg \rho \leqq \frac{\pi}{\mu}\right\}
$$

Let $\omega=e^{\pi i / \mu}$. Then in the first case, for $\arg \rho=0$

$$
\left.\Re\left(\rho \omega^{\mu / 2}\right)=\Re(\rho \omega)^{-\mu / 2}\right)=0 .
$$

In the second case

$$
\Re\left(\rho \omega^{(\mu-1) / 2}\right)=\mathfrak{K}\left(\rho \omega^{-(\mu+1) / 2}\right)=0
$$

for $\arg \rho=\pi / 2 \mu$. Suppose the indexing is arranged so that in the first case

$\omega_{1}=\omega^{-\mu / 2}, \omega_{2}=\omega^{-\mu / 2+1}, \cdots, \omega_{\mu}=\omega^{\mu / 2-1}, \omega_{\mu+1}=\omega^{\mu / 2}, \omega_{\mu+2}=\omega^{\mu / 2+1}, \cdots, \omega_{2 \mu}=\omega^{3 \mu / 2-1}$

and in the second,

$$
\omega_{1}^{\prime}=\omega^{-\mu / 2-1 / 2}, \omega_{2}^{\prime}=\omega^{-\mu / 2+1 / 2}, \cdots, \omega_{\mu}^{\prime}=\omega^{\mu / 2-3 / 2}, \omega_{\mu+1}^{\prime}=\omega^{\mu / 2-1 / 2}, \cdots, \omega_{2 \mu}^{\prime}=\omega^{3 \mu / 2-3 / 2} .
$$

Upon bringing $e^{\rho \omega_{k}}$ out of the determinant wherever $\Re\left(\rho \omega_{k}\right)>0$, one has

$$
\delta(\rho)=\prod_{i=1}^{\mu} \rho^{k_{i}+l_{i}} \prod_{i=2}^{\mu+1} e^{\rho \omega_{i}} \delta^{\prime}(\rho)
$$

where $\delta^{\prime}(\rho)$ has the appearance 


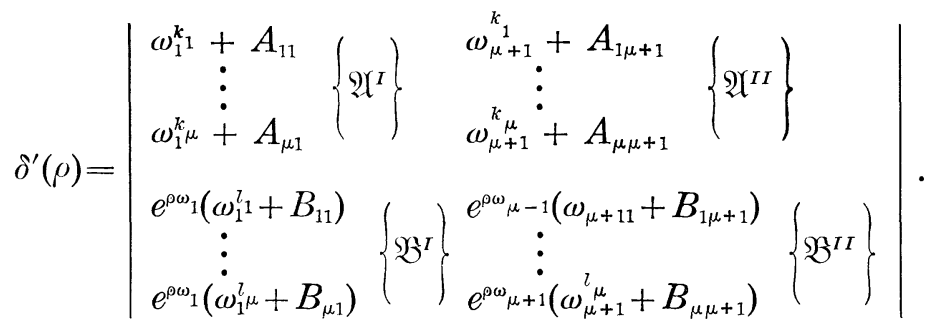

Here, $\mathfrak{Q}^{I}$ and $\mathfrak{B}^{I I}$ are matrices consisting of $\mu-1$ columns and $\mu$ rows all of whose terms have for a factor an exponential term with negative real part. Asymptotically, these matrices are therefore negligible.

$$
\begin{aligned}
& \mathfrak{V} I^{I I}=\left\{\begin{array}{c}
\omega_{\mu+2}^{{ }^{k}}+A_{1 \mu+2} \cdots \omega_{2 \mu}^{{ }_{1}}+A_{12 \mu} \\
\vdots \\
\omega_{\mu+2}^{{ }_{\mu}{ }_{\mu}+} A_{\mu \mu+2} \cdots \omega_{2 \mu}^{{ }_{\mu}}+A_{\mu \nu \mu}
\end{array}\right\} \\
& \mathfrak{V}^{I}=\left\{\begin{array}{c}
e^{\rho \omega_{2}}\left(\omega_{2}^{l}+B_{12}\right) \cdots e^{\rho \omega_{\mu}}\left(\omega_{\mu}^{l}+B_{1 \mu}\right) \\
\vdots \\
e^{\rho \omega_{2}}\left(\dot{\omega}_{2}^{l} \mu+B_{\mu 2}\right) \cdots e^{\rho \omega_{\mu}}\left(\omega_{\mu}^{l}{ }_{\mu}+B_{\mu \mu}\right)
\end{array}\right\} .
\end{aligned}
$$

Thus

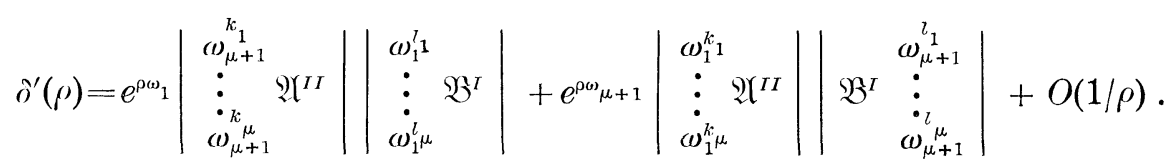

Only the case in which $\mu$ is even shall be considered explicitly since the treatment for $\mu$ odd is completely analagous. First note that

$$
\omega_{1}=e^{-\pi i / 2}=-i
$$

and

$$
\omega_{\mu+1}=e^{\pi i / 2}=i
$$

Thus $\omega_{1}=-\omega_{\mu+1}$. Now let $x_{i} \equiv \omega^{k_{i}}$ and $y_{i} \equiv \omega^{i_{i}}$. The conditions that $k_{1}>k_{2}>\cdots>k_{\mu}$ and $l_{1}>l_{2}>\cdots l_{\mu}$ and the fact that $\omega=e^{i \pi / \mu}$ is a primitive root of unity imply that $x_{i} \neq x_{1}, y_{i} \neq y_{1}$, for $i \neq j$. Recall that by the arrangement of the indices, $\omega_{s}=\omega^{(-\mu / 2)+s-1}=\omega^{-\mu / 2} \omega^{s-1}$. Therefore,

$$
\omega_{s}^{k_{i}}=\omega^{(-\mu / 2) k_{i}} \omega^{(s-1) k_{i}}=x_{i}^{-\mu / 2} x_{i}^{(s-1)}
$$

and

$$
\omega_{s}^{l}=\omega^{(-\mu / 2) l_{i}^{(s-1) l}}{ }_{i}=y_{i}^{-\mu / 2} y_{i}^{(s-1)} .
$$

Using now the explicit representation of $\mathfrak{U}^{I I}$ and $\mathfrak{V}^{I}$ given previously, but taking only zero order terms into account, one has 


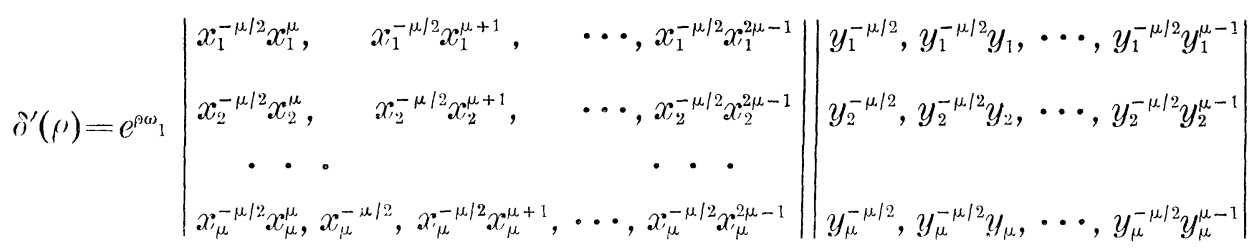

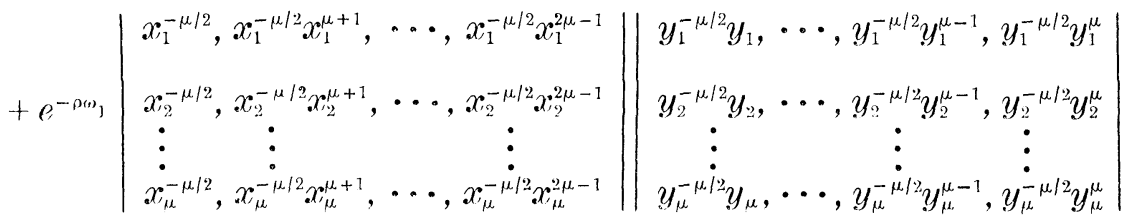

$$
\begin{aligned}
& +O(1 / \rho) \text {. }
\end{aligned}
$$

By bringing common factors outside the determinant, one can simplify the expression for $\delta^{\prime}(\rho)$.

$$
\begin{aligned}
& \sigma^{\prime}(o)=e^{p(t+1} \prod_{i=1}^{\mu} x_{i}^{\mu / 2} y_{i}^{-\mu l:}\left|\begin{array}{cccc}
1 & x_{1} & \cdots & x_{1}^{\mu-1} \\
1 & x_{2} & \cdots & x_{*}^{\mu-1} \\
\vdots & \vdots & & \vdots \\
1 & x_{\mu} & \cdots & x_{\mu}^{\mu-1}
\end{array}\right|\left|\begin{array}{cccc}
1 & y_{1} & \cdots & y_{1}^{\mu-1} \\
1 & y_{2} & \cdots & y_{2}^{\mu-1} \\
\vdots & \vdots & & \vdots \\
1 & y_{\mu} & \cdots & y_{\mu}^{\mu-1}
\end{array}\right| \\
& +e^{-\rho(1)} \prod_{i=1}^{\mu} x_{i}^{-\mu / 2} y_{1}^{-\mu / 2 / 2}\left|\begin{array}{cccc}
1 & x_{1}^{\mu+1} & \cdots & x_{1}^{2 \mu-1} \\
1 & x_{2}^{\mu+1} & \cdots & x_{2}^{2 \mu-1} \\
\vdots & \vdots & & \vdots \\
1 & x_{\mu}^{\mu+1} & \cdots & x_{\mu}^{2 \mu-1}
\end{array}\right|\left|\begin{array}{cccc}
1 & y_{1} & \cdots & y_{1}^{\mu-2}, y_{1}^{\mu-1} \\
1 & y_{2} & \cdots & y_{2}^{\mu-2}, y_{2}^{\mu-1} \\
\vdots & \vdots & & \vdots \\
1 & y_{\mu} & \cdots & y_{\mu}^{\mu-2}, \\
\vdots & y_{\mu}^{\mu-1}
\end{array}\right|+O(1 / \rho) \text {. }
\end{aligned}
$$

The first determinantal factor of the second term above can be treated by noting that $1=x_{i}^{2 \mu}$ and switching columns $\mu-1$ times and then bringing the factor $x_{i}^{\mu}$ outside. These manipulations yield

$$
\begin{aligned}
\gamma^{\prime}(\rho)= & e^{\rho(1) 1} \prod_{i=1}^{\mu} x_{i}^{\mu / 2} y_{i}^{-\mu / 2}\left|\begin{array}{cccc}
1 & x_{1} & \cdots & x_{1}^{\mu-1} \\
1 & x_{2} & \cdots & x_{2}^{\mu-1} \\
\vdots & & \vdots \\
1 & x_{\mu} & \cdots & x_{\mu}^{\mu-1}
\end{array}\right|\left|\begin{array}{cccc}
1 & y_{1} & \cdots & y_{1}^{\mu-1} \\
1 & y_{2} & \cdots & y_{2}^{\mu-1} \\
\vdots & \vdots & & \vdots \\
1 & y_{\mu} & \cdots & y_{\mu}^{\mu-1}
\end{array}\right| \\
& -e^{-\rho()_{1}} \prod_{i=1}^{\mu} x_{i}^{\mu / 2} y_{i}^{\mu / 2}\left|\begin{array}{cccc}
1 & x_{1} & \cdots & x_{1}^{\mu-1} \\
1 & x_{2} & \cdots & x_{2}^{\mu-1} \\
& \vdots & & \\
1 & x_{\mu} & \cdots & x_{\mu}^{\mu-1}
\end{array}\right|\left|\begin{array}{cccc}
1 & y_{1} & \cdots & y_{1}^{\mu-1} \\
1 & y_{2} & \cdots & y_{2}^{\mu-1} \\
& \vdots & & \vdots \\
1 & y_{\mu} & \cdots & y_{\mu}^{\mu-1}
\end{array}\right|+O(1 / \rho) .
\end{aligned}
$$

Now note that the determinants involved in the above expression are Vandermonde determinants. But such determinants do not vanish provided only the entries $\left(x_{1}, x_{*}, \cdots, x_{\mu}\right)$ or $\left(y_{1}, y_{2}, \cdots, y_{\mu}\right)$ are distinct. That this is the case was demonstrated above. Therefore, the given 
boundary conditions are regular in the sense of Birkhoff since in the equation

$$
\delta^{\prime}(\rho)=\theta_{0}+\theta_{1} e^{\rho \omega_{1}}+\theta_{2} e^{-\rho \omega_{1}}
$$

not both $\theta_{1}$ and $\theta_{2}$ vanish. Tamarkin [11] who examined "separated" boundary conditions failed to reach tbis general conclusion. By including common factors in the term $O(1 / \rho)$, the equation $\delta^{\prime}(\rho)=0$ can now be written in the form

$$
e^{\rho \omega_{1}}-e^{-\rho \omega_{1}} \prod_{i=1}^{\mu} y_{i}^{\mu}+O(1 / \rho)=0
$$

But

$$
y_{k}^{\mu}=\omega^{l} k_{\mu}=e^{(\pi i / \mu) l_{k}^{\mu}}=e^{\mu i l_{k}}=(-1)^{l} k_{k} .
$$

Hence, on multiplying by $e^{-\rho \omega_{1}}$, one obtains

$$
e^{2 \rho \omega_{1}}=(-1)^{\Sigma_{k=1}^{\mu} l_{k}}+O(1 / \rho)
$$

or

$$
e^{2 \rho i}=(-1)^{\Sigma_{k=1}^{\mu}{ }_{k}{ }_{k}}+O(1 / \rho) .
$$

On taking square roots of both members, one finds that

$$
e^{\rho i}= \pm i(1+O(1 / \rho))
$$

or

$$
e^{\rho t}= \pm(1+O(1 / \rho)) .
$$

Taking logarithms of both members and noting that

$$
\log (1+O(1 / \rho))=O(1 / \rho)
$$

results in the expressions

$$
\rho_{I k}=\pi / 2+2 \pi k+O(1 / \rho)
$$

$$
\rho_{I I k}=\pi / 2+2 \pi k+O(1 / \rho)
$$

or

$$
\begin{gathered}
\rho_{I k}=2 \pi k+O(1 / \rho) \\
\rho_{I I k}=\pi+2 \pi k+O(1 / \rho) .
\end{gathered}
$$

By neglecting the terms of order $1 / \rho$ first estimates may be obtained which may then be inserted in (9) or (10) with the following results: 


$$
\rho_{I:}=(2 \pi k)\left\{1+\frac{1}{4 k}+\frac{E_{I}(k)}{k^{2}}\right\}
$$

(11)

$$
\rho_{I I k}=(2 \mu k)\left\{1-\frac{1}{4 k}+\frac{E_{I I}(k)}{k^{2}}\right\}
$$

or

$$
\rho_{I k}=(2 \pi k)\left\{1+\frac{E_{I}(k)}{k^{2}}\right\}
$$

(12)

$$
\rho_{I I k}=(2 \pi k)\left\{1+\frac{1}{2 k}+\frac{E_{I I}(k)}{k^{2}}\right\}
$$

where the $E_{I}(k)$ and $E_{I I}(k)$ represent bounded functions of $k$.

It should be noted that (11) and (12) are valid not only in the case in which $\mu$ is even, but also when $\mu$ is odd.

Reverting to the $\lambda$-plane one finds that

$$
\lambda_{I k}=(2 \pi k)^{2 \mu}\left\{1+\frac{\mu}{2 k}+\frac{E_{I}(k)}{k^{2}}\right\}
$$

(13)

$$
\lambda_{I I k}=(2 \pi k)^{2 \mu}\left\{1-\frac{\mu}{2 k}+\frac{E_{I I}(k)}{k^{2}}\right\}
$$

or

$$
\lambda_{I k}=(2 \pi k)^{2 \mu}\left\{1+\frac{E_{I}(k)}{k^{2}}\right\}
$$

$$
\lambda_{I I k}=(2 \pi k)^{2 \mu}\left\{1+\frac{\mu}{k}+\frac{E_{I I}(k)}{k^{2}}\right\} .
$$

Since the zeroes of $\Delta(\lambda)$ furnish the poles of the Green's function, one sees that all except a finite set of characteristic values of $T$ are simple poles of the resolvent. This does not, however, assert anything about the number of linearly independent characteristic functions associated with each characteristic value. This matter will be dealt with below.

From (13) and (14) one obtains expressions for the distance separating the points of the spectrum $\sigma(T)$. 


$$
\begin{aligned}
&\left|\lambda_{I k}-\lambda_{I I k}\right|=(2 \pi k)^{2 \mu}\left\{\frac{\mu}{k}+\frac{E(k)}{k^{2}}\right\} \\
&=(2 \pi)^{2 \mu} k^{2 \mu-1}\left\{\mu+\frac{E(k)}{k}\right\} . \\
&\left|\lambda_{I k+1}-\lambda_{I k}\right|=(2 \pi)^{\mu} k^{2 \mu-1}\left\{2 \mu+\frac{E(k)}{k}\right\}
\end{aligned}
$$

so that in any case, for $\tau>1 / 2 \mu-1$

$$
\sum_{k=1}^{\infty} d_{k}^{-\tau}<\infty
$$

This verifies that Condition B is satisfied.

In view of a prior remark it is merely necessary to exhibit a nonvanishing first minor of $\Delta(\lambda)$ in order to permit the conclusion that all but a finite number of characteristic values are simple. Moreover, since $\Delta(\lambda)=F(\rho) \delta^{\prime}(\rho)$ where $F(\rho) \neq 0$ it is sufficient to find a nonvanishing first minor of $\delta^{\prime}(\rho)$.

Reverting to the expression for $\delta^{\prime}(\rho)$ and singling out the minor of the element in the first column and $2 \mu$ th row results in the exhibit:

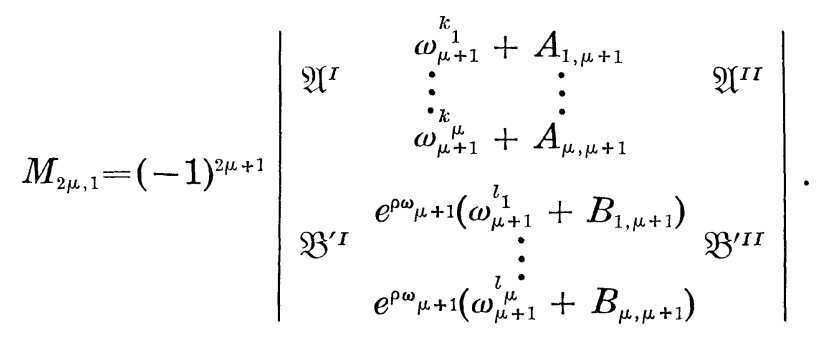

Here $\mathfrak{B}^{\prime I}$ and $\mathfrak{B}^{\prime I I}$ are obtained from $\mathfrak{B}^{I}$ and $\mathfrak{B}^{I I}$ by deleting the last row in each of these matrices. On expanding $M_{2 \mu, 1}$ in terms of the $\mu \times \mu$ minors occupying the first $\mu$ rows and their complements and noting that all the terms of $\mathfrak{U}^{I}$ and $\mathfrak{B}^{\prime I I}$ are negligible in view of the fact that each has an exponential factor with negative real part, one has

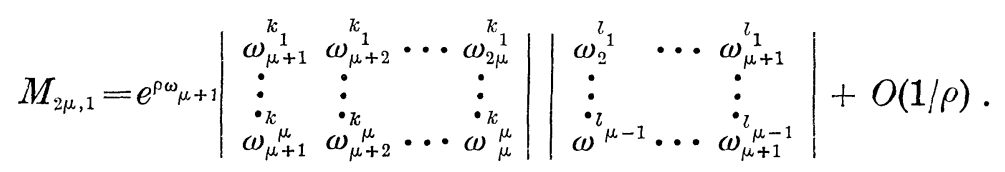

In the previously employed notation, $x_{i}=\omega^{k_{i}}, y_{i}=\omega^{l_{i}}$, one can write $M_{2 \mu, 1}$ in the form:

$$
M_{2 \mu, 1}=e^{\rho \omega_{\mu+1}} \prod_{i=1}^{\mu} x_{i}^{\mu / 2}\left|\begin{array}{cccc}
1 & x_{1} & \cdots & x_{1}^{\mu-1} \\
1 & x_{2} & \cdots & x_{2}^{\mu-1} \\
\vdots & \vdots & & \vdots \\
1 & x_{\mu} & \cdots & x_{\mu}^{\mu-1}
\end{array}\right| \prod_{i=1}^{\mu-1} y_{i}^{\mu / 2}\left|\begin{array}{cccc}
1 & y_{1} & \cdots & y_{1}^{\mu-1} \\
1 & y_{2} & \cdots & y_{2}^{\mu-1} \\
\vdots & \vdots & & \vdots \\
1 & y_{\mu-1} & \cdots & y_{\mu-1}^{\mu-1}
\end{array}\right|,
$$


Again, the Vandermonde determinants appearing above do not vanish because the $x_{i}$ and the $y_{i}$ are distinct. Upon using the previously derived expressions for $\rho_{k}$, one sees that $\left|e^{\rho{ }^{\omega} \mu_{\mu+1}}\right| \stackrel{k}{\rightarrow} 1$. Hence, it follows that $M_{2 \mu, 1} \neq 0$ except, possibly, for a finite number of indices $k$, and thus all except possibly a finite number of characteristic values are simple.

In order to show that $T$ is spectral, it is necessary at this point merely to establish a uniform bound on the spectral resolution $E(e)$ of $T$. But because $T$ is regular this is tantamount to giving a uniform bound for sums

$$
\sum_{k \in J} E\left(\lambda_{k}\right)
$$

whenever $J$ is a finite set of indices. In establishing such a bound, the finite set of $\left\{\lambda_{k}\right\}$ which are multiple poles of the resolvent or multiple characteristic values cause no difficulty. Therefore, it shall be supposed that $E\left(\lambda_{k}\right)$ projects onto a one-dimensional range. One can construct $E\left(\lambda_{k}\right)$ explicitly by drawing on Lemma 12 of J. Schwartz which states:

" Let $E$ be a projection of $B$-space $X$ onto a finite dimensional range, and let $E^{*}: X^{*} \rightarrow X^{*}$ be its adjoint. Then if $\varphi_{1}, \cdots, \varphi_{n}$ is a basis of $E X$, we can find a unique basis of $\psi_{1}^{*}, \cdots, \psi_{n}^{*}$ of $E^{*} X^{*}$ such that $\psi^{*}\left(\varphi_{j}\right)=\delta_{i, j}$, and then

$$
E f=\sum_{i=1}^{n} \varphi_{i} \psi_{i}^{*}(f)
$$

for any $f \in X$.

Now let $\varphi_{m}(x)$ be the $m$ th characteristic function of $T$, and $\psi_{m}(x)$ the corresponding $m$ th characteristic function of $T^{*}$. Then

$$
E\left(\lambda_{m}\right) f=\frac{\int_{0}^{1} \varphi_{m}(x) \psi_{m}\left({ }^{\prime \prime}\right) f(y) d y}{\int_{0}^{1} \varphi_{m}(x) \overline{\psi_{m}(x)} d x} .
$$

Now suppose that

$$
\begin{aligned}
& \varphi_{m}(x)=\theta_{m}(x)+\frac{1}{m} K_{1}(m, x) \\
& \phi_{m}(x)=\theta_{m}(x)+\frac{1}{m} K_{2}(m, x)
\end{aligned}
$$

where $K_{1}(m, x)$ and $K_{2}(m, x)$ are uniformly bounded in $m$. Then $\varphi_{m}(x) \overline{\psi_{m}}(y)=\theta_{m}(x) \bar{\theta}_{m}(y)+\frac{\theta_{m}(x) \bar{K}_{2}(m, y)}{m}+\frac{K_{1}(m, x) \overline{\theta_{m}(y)}}{m}+\frac{K_{1}(m, x) \bar{K}_{2}(m, y)}{m^{2}}$ 
and

$$
\int_{0}^{1} \varphi_{m}(x) \psi_{m}(x) d x=\left(\varphi_{m}, \psi_{m}\right)=\left\|\theta_{m}\right\|^{2}+\frac{1}{m}\left(\theta_{n}, K_{2}\right)+\frac{1}{m}\left(K_{1}, \theta_{m}\right)+\frac{1}{m^{2}}\left(K_{1}, K_{2}\right) .
$$

Upon inserting these expansions in the expression for $E\left(\lambda_{m}\right) f$ above, we get

$$
\begin{aligned}
E\left(\lambda_{m}\right) f= & \frac{\int_{0}^{1} \theta_{m}(x) \overline{\theta_{m}(y)} f(y) d y}{\left\|\theta_{m}\right\|^{2}}+\frac{C_{1}(m)}{m} \frac{\int_{0}^{1} \theta_{m}(x) \overline{K_{2}(m, y)} f(y) d y}{\left\|\theta_{m}\right\|^{2}} \\
& +\frac{C_{2}(m)}{m} \frac{\int_{0}^{1} K_{1}(m, x) \overline{\theta_{m}(y)} f(y) d y}{\left\|\bar{\theta}_{m}\right\|^{2}}+\frac{C_{3}(m)}{m^{2}} \int_{0}^{1} K_{1}(m, x) \overline{K_{2}}(m, y) f(y) d y \\
= & \hat{E}_{m} f+\frac{1}{m} \hat{E}_{m} A_{m} f+\frac{1}{m} B_{m} \hat{E}_{m} f+\frac{K_{m} f}{m^{2}}
\end{aligned}
$$

where $\hat{E}_{m}$ is a Hermitian projection since its norm is unity and it is an integral operator with symmetric kernel and $A_{n}, B_{m}, K_{m}$ are multiplication operators that are uniformly bounded in $m$.

Now if $J$ is a finite set of integers,

$$
\begin{aligned}
\left\|\sum_{n \in J} E\left(\lambda_{n}\right)\right\| \leqq & \left\|\sum_{n \in J} \hat{E}_{n}\right\|+\left\|\sum_{n \in J} \frac{1}{n} \hat{E}_{n} A_{n}\right\| \\
& +\left\|\sum_{n \in J}{ }^{1} B_{n} \hat{E}_{n}\right\|+\left\|\sum_{n \in J} \frac{1}{n^{2}} K_{n}\right\| .
\end{aligned}
$$

The first term is bounded by 1 because of the Hermitian character of the idempotents. Applying Lemma 3 to the second and third terms yields the bounds

$$
\sup _{n}\left\|A_{n}\right\| \sum_{n=1}^{\infty} \frac{1}{n^{2}}
$$

and

$$
\sup _{n}\left\|B_{n}\right\| \sum_{n=1}^{\infty} \frac{1}{n^{2}}
$$

For the fourth term one has the bound

$$
\sup _{n}\left\|K_{n}\right\| \sum_{n=1}^{\infty} \frac{1}{n^{2}} .
$$

So that, granting the above representation for the characteristic func- 
tions of the operator $T$ and the adjoint $T^{*}$, one may draw the conclusion that $T$ is spectral.

In order to exhibit $\varphi_{m}(x)$ and $\psi_{m}(x)$ in the forms (15) and (16) it is necessary once more to resort to an asymptotic development. (cf. p. )

$$
\begin{aligned}
\varphi_{m}(x) & =\sum_{i=1}^{2 \mu} C_{i} u_{i}(x) \quad u_{i}(x)=e^{\rho_{m} \omega_{i} x} \\
0 & =\sum_{i=1}^{2 \mu} C_{i} A_{j}\left(u_{i}\right) \\
0 & =\sum_{i=1}^{2 \mu} C_{i} B_{j}\left(u_{i}\right)
\end{aligned}
$$$$
j=1,2, \cdots, \mu \text {. }
$$

From the compatibility of equations (17) it follows that $C_{i}$ is proportional to the minor $M_{\mu+1, i}$ of the element in the $\mu+1$ st column and the $i$ th row of the matrix. This gives then the representation

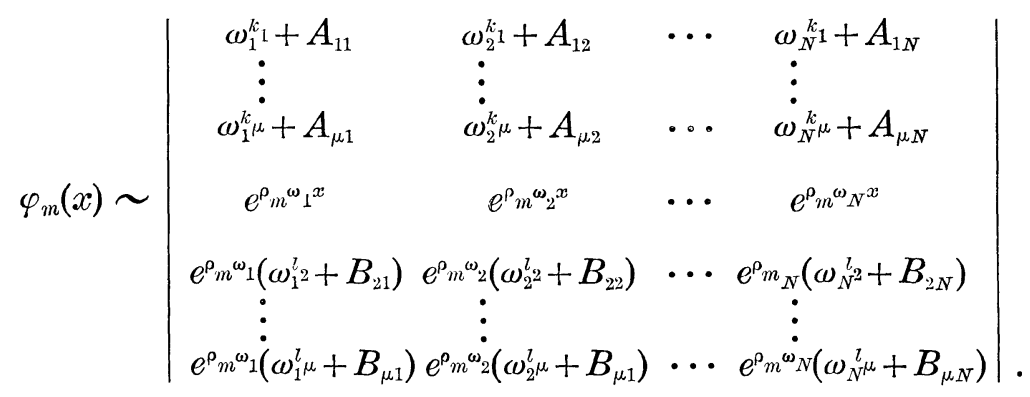

Here and in similar expressions to follow, proportionality factors are freely discarded. Since the above determinant closely resembles $\delta^{\prime}(\rho)$, essentially the same techniques that were successful before shall be applied again. For $k=2,3, \cdots, \mu$ we have $\Re\left(\rho_{m} \omega_{k}\right)>0$. Bring $e^{\rho} m^{\omega_{k}}$ outside the determinant.

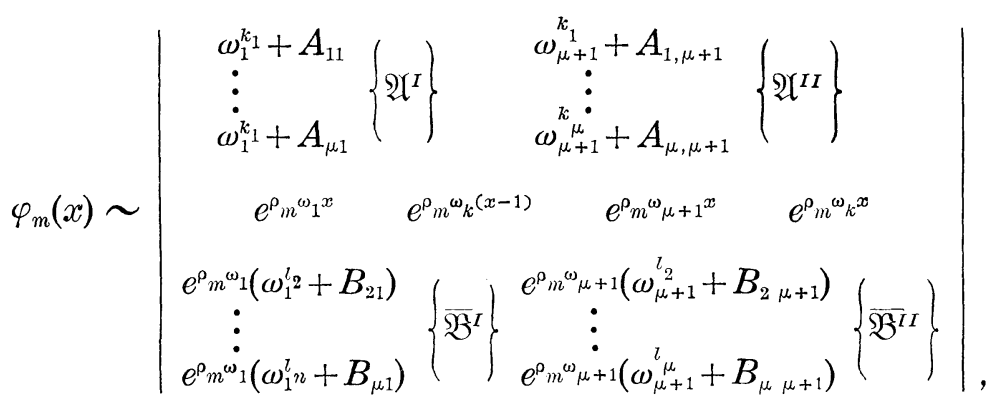

The entries of the matrices $\mathfrak{A}^{I}$ and $\overline{\mathfrak{B}}^{I I}$ are all negligible for large $k$. Expanding the above expression one obtains 


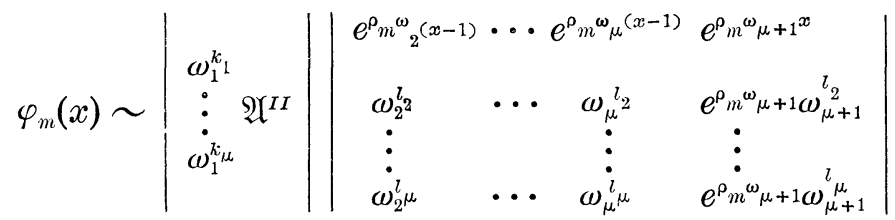

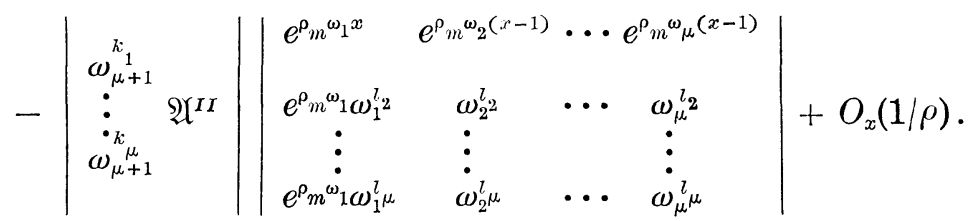

Recall that the two determinants involving $\mathfrak{A}^{I I}$ are proportional to lowest order in $1 / \rho$ and that the factor of proportionality is \pm 1 . Hence, on incorporating this factor in $O(1 / \rho ; x)$ and bringing $e^{\rho_{m} \omega_{\mu+1}}$ and $e^{\rho_{m} \omega_{1}}$ outside the determinants one has:

$$
\begin{aligned}
& \varphi_{m}(x) \sim e^{-\rho_{m} \omega_{1}}\left|\begin{array}{cccc}
e^{\rho_{m} \omega_{2}(x-1)} & \cdots & e^{\rho_{m} \omega_{\mu}(x-1)} & e^{\rho_{m} \omega_{\mu+1}(x-1)} \\
\omega_{2}^{l} & \cdots & \omega_{\mu}^{l} & \omega_{\mu+1}^{l_{2}} \\
\vdots & & \vdots & \vdots \\
\omega_{2}^{l} l^{2} & \cdots & \omega_{\mu}^{l} & \omega_{\mu+1}^{2}
\end{array}\right| \\
& \pm e^{\rho_{m} \omega_{1}}\left|\begin{array}{cccc}
e^{\rho_{m} \omega_{1}(x-1)} & e^{\rho} m_{2}^{\omega_{2}}(x-x) & \cdots & e^{\rho_{m} \omega_{\mu}(x-1)} \\
\omega_{12}^{l_{2}} & \omega_{2}^{l_{2}} & \cdots & \omega_{\mu^{2}}^{l^{2}} \\
\vdots & \vdots & & \vdots \\
\omega_{1}^{l_{\mu}} & \omega_{2}^{l^{l} \mu} & \cdots & \omega_{\mu}^{l_{\mu}}
\end{array}\right|+O_{x}(1 / \rho) .
\end{aligned}
$$

It should be noted that except at $x=1$, the terms $\epsilon^{p} m^{\omega_{k}}(-1) x, k \neq 1$ are negligible asymptotically since $\Re\left(\rho_{m} \omega_{k}(x-1)\right)<0$. Now using the previously obtained asymptotic expressions for $\rho_{m}$, one finds that for $x \neq 1$

$$
\begin{aligned}
& \varphi_{m I}(x) \sim e^{-2 \pi i(m+1 / 4) x}\left|\begin{array}{ccc}
\omega_{2}^{l} 2 & \cdots & \omega_{\mu}^{l_{2}} \\
\vdots & & \vdots \\
\omega_{2}^{l} \mu & \cdots & \omega_{\mu}^{l} \mu
\end{array}\right| \\
& -e^{2 \pi i(m+1 / 4) x}\left|\begin{array}{ccc}
\omega_{2}^{l} & \cdots & \omega_{\mu}^{l}{ }^{2} \\
\vdots & & \\
\omega_{2}^{l} \mu & \cdots & \omega_{\mu}^{l} \mu
\end{array}\right|+O(1 / m ; x) \\
& \varphi_{m I I}(x) \sim e^{-2 \pi i(m-1 / 4) x}\left|\begin{array}{ccc}
\omega_{2}^{l_{2}} & \cdots & \omega_{\mu}^{l_{2}} \\
\vdots & & \\
\omega_{2}^{l_{\mu}} & \cdots & \omega_{\mu}^{l_{\mu}}
\end{array}\right| \\
& -e^{2 \pi i(m-1 / 4) x}\left|\begin{array}{ccc}
\omega_{2}^{l} & \cdots & \omega_{\mu^{2}}^{l} \\
\vdots & & \\
\omega_{2}^{l} \mu & \cdots & \omega_{\mu}^{l} \mu
\end{array}\right|+O(1 / m ; x)
\end{aligned}
$$


or else

$$
\begin{aligned}
& \varphi_{m I}(x) \sim e^{-2 \pi i m x}\left|\begin{array}{ccc}
\omega_{2}^{l} & \cdots & \omega_{\mu^{\mu}}^{l} \\
\vdots & & \\
\omega_{2}^{l} & \cdots & \omega_{\mu}^{l}
\end{array}\right| \\
& -e^{2 \pi i m x}\left|\begin{array}{ccc}
\omega_{2}^{l_{2}} & \cdots & \omega_{\mu^{2}}^{l^{2}} \\
\vdots & & \\
\omega_{2}^{l}{ }^{l} & \cdots & \omega_{\mu^{\mu}}^{l}
\end{array}\right|+O(1 / m ; x) \\
& \varphi_{m I I}(x) \sim e^{-2 \pi i(m+1 / 2) x}\left|\begin{array}{ccc}
\omega_{2}^{l^{2}} & \cdots & \omega_{\mu^{2}}^{l_{2}} \\
\vdots & & \\
\omega_{2}^{l_{k}} k & \cdots & \omega_{\mu}^{l^{\mu}}
\end{array}\right| \\
& -e^{2 \pi i(m+1 / 2) x}\left|\begin{array}{ccc}
\omega_{2}^{l} & \cdots & \omega_{\mu^{2}}^{l^{2}} \\
\vdots & & \vdots \\
\omega_{2}^{l} & \cdots & \omega_{\mu^{\mu}}^{l}
\end{array}\right|+O(1 / m ; x) .
\end{aligned}
$$

On incorporating common factors that are uniformly bounded with respect to $m$ into the terms $O(1 / m ; x)$ one has

$$
\varphi_{m I}(x)=\sin 2 \pi\left(m+\frac{1}{4}\right) x+\frac{K_{1 I}(m, x)}{m}
$$

(18)

$$
\varphi_{m I I}(x)=\sin 2 \pi\left(m-\frac{1}{4}\right) x+\frac{K_{1 I I}(m, x)}{m}
$$

or

$$
\varphi_{m I}(x)=\sin 2 \pi m x+\frac{K_{11}(m, x)}{m}
$$

(19)

$$
\varphi_{m I I}(x)=\sin 2 \pi\left(m+\frac{1}{2}\right) x+\frac{K_{1 I I}(m, x)}{m} .
$$

Thus the characteristic functions of $T$ have been brought into the desired form. Note, however, that since $\tau=d^{N} / d x^{N}$ is formally selfadjoint, $T$ and $T^{*}$ differ only in the boundary conditions. But it is a simple matter to see that the boundary conditions of $T^{*}$ will again be of the "separated" type (cf. [7, p. 186]) and that therefore all the developments leading to an asymptotic expression for $\psi_{m I}$ and $\psi_{m I I}$ will be the same as those that served to find (18) and (19). Now note that the first terms in (18) and (19) in no way reflect the quantities occurring in the boundary conditions. Therefore it may be concluded that 


$$
\psi_{m I}(x)=\sin 2 \pi\left(m+\frac{1}{4}\right) x+\frac{K_{2 I}(m, x)}{m}
$$

(20)

$$
\psi_{m I I}(x)=\sin 2 \pi\left(m-\frac{1}{4}\right) x+\frac{K_{2 I I}(m, x)}{m}
$$

or

$$
\psi_{m I}(x)=\sin 2 \pi m x+\frac{K_{2 I}(m, x)}{m}
$$

$$
\psi_{m I I}(x)=\sin 2 \pi\left(m+\frac{1}{2}\right) x+\frac{K_{2 I I}(m, x)}{m} .
$$

By what has preceded, then, it may be concluded that $T$ is spectral.

To complete the verification of Condition A for $T$ is still necessary to show that

$$
\sum_{k=1}^{\infty} E\left(\lambda_{k}\right)=I
$$

To this end note that

$$
\lim _{m \rightarrow \infty}\left\|\left(I-\sum_{k=m}^{\infty} E\left(\lambda_{k}\right)\right)-\left(I-\sum_{k=m}^{\infty} \hat{E}_{k}\right)\right\|=0
$$

so that in virtue of the fact that $\hat{E}_{k}$ is Hermitian and the above cited lemma of J. Schwartz and F. Wolf, the range of

$$
I-\sum_{k=n}^{\infty} E\left(\lambda_{k}\right)
$$

for sufficiently large $m$ is finite dimensional. But in his Lemma 15 J. Schwartz asserts that

$$
S_{\infty} \equiv\left\{f \mid E\left(\lambda_{k}\right) f=0,0<k<\infty\right\}
$$

is either infinite dimensional or else the null space. But since

$$
S_{\infty} \leqq \operatorname{range}\left(I-\sum_{k=m}^{\infty} E\left(\lambda_{k}\right)\right)
$$

the above implies that $S_{\infty}$ is finite dimensional and hence consists of the null vector alone.

It remains to verify the special hypothesis placed on the spectrum of $T$ in Theorem 1 and embodied in the requirement that for all sufficiently large $N$, for all 


$$
\begin{gathered}
\lambda \in C_{N} \equiv\left\{\lambda|| \lambda-\lambda_{N} \mid=\frac{1}{3} d_{N}\right\}, \\
\max _{z_{k} \in \sigma(T)} \frac{\left|z_{k}\right|^{\nu}}{\left|\lambda-z_{k}\right|}<\frac{C}{d_{N}^{\tau / 2}}
\end{gathered}
$$

where

$$
d_{N}=\operatorname{dist}\left[\lambda_{N}, \sigma(T) \sim\left\{\lambda_{N}\right\}\right]
$$

First observe that if $N=k$,

$$
\frac{\left|z_{k}\right|^{\nu}}{\left|\lambda-z_{k}\right|}=\frac{\left|\lambda_{N}\right|^{\nu}}{d_{N}}
$$

and if $N \neq k$

$$
\begin{aligned}
\frac{\left|z_{k}\right|^{\nu}}{\left|\lambda_{N}-z_{k}\right|} & =\frac{1}{\left|D_{N k}\right|^{1-\nu}}\left|\frac{\lambda_{N}}{D_{N k}}+e^{i \varphi}\right| \leqq \frac{1}{\left|D_{N k}\right|^{1-\nu}}\left\{\left|\frac{\lambda_{N}}{D_{N k}}\right|+1\right\}^{\nu} \\
& \leq 1 \\
& \left.\leq \frac{\left|\lambda_{N}\right|}{d_{N}^{1-\nu}}+1\right\}^{\nu} \leqq \frac{\left\{\left|\lambda_{N}\right|+1\right\}^{\nu}}{d_{N}} \\
D_{N k} & \equiv\left(z_{k}-\lambda_{N}\right) e^{-i \varphi} .
\end{aligned}
$$

In any case, therefore, there exists a $C$ such that

$$
\max _{z_{k} \in \sigma(T)} \frac{\left|z_{k}\right|^{\nu}}{\left|\lambda-z_{k}\right|}<C \frac{\left|\lambda_{N}\right|^{\nu}}{d_{N}} .
$$

Now recall that according to the previously obtained asymptotic formulas, $\lambda_{N} \sim N^{2 \mu}$ and $d_{N} \sim N^{2 \mu-1}$. Hence

$$
\frac{\left|\lambda_{N}\right|^{\nu}}{d_{N}} \sim N^{2 \mu(\nu-1)+1} .
$$

Convergence of

$$
\sum_{k=1}^{\infty} \frac{1}{d_{k}^{\tau}}
$$

is assured for $\tau>1 / 2 \mu-1$. It is thus required that $2 \mu(\nu-1)+1<-1 / 2$. This requirement is satisfied by taking $\nu<(\mu-3 / 4) / \mu$ and, a fortiori, by the choice $\nu=(2 \mu-2) / 2 \mu$.

Finally. it is necessary to determine the class of operators $S$ for which $\mathscr{D}(S) \supset \mathscr{S}\left(T^{\nu}\right)$. To this end it shall be shown first that if $f(x) \in \mathscr{C}\left(T^{\nu}\right)$, then $f(x)$ is $\nu=2 \mu-2$ times differentiable. Suppose $f \in \mathscr{D}\left(T^{\nu}\right)$. Then 


$$
f(x)=\sum_{k=1}^{\infty} E\left(\lambda_{k}\right) f
$$

If this series is differentiated termwise $2 \mu-1$ times one has formally,

$$
\begin{aligned}
\sum_{k=1}^{\infty}(-1)^{\mu-1}(2 \pi)^{2 \mu-2}(k \pm 1 / 2)^{2 \mu-2} & \\
& \times\left\{\int_{0}^{1} \sin 2 \pi(k \pm 1 / 4) x \sin 2 \pi(k \pm 1 / 4) y f(y) d y+\frac{K \pm(x, f)}{k}\right\} .
\end{aligned}
$$

But this expansion converges for $f \in \mathscr{D}\left(T^{\nu}\right)$ almost everywhere to $f^{(2 \mu-2)}(x)$. Now let $S$ be any closed operator whose domain consists of $2 \mu-2$ times differentiable functions on the closed interval $(0,1)$ such that the $(2 \mu-2) n d$, derivative is square-integrable. Theorem 1 applies.

Thus in conclusion one has the following.

THEOREM 3. Let $T$ be the operator $d^{2 \mu} / d x^{2 \mu}$ with boundary conditions

$$
\begin{array}{rr}
A_{i}(f)=f^{\left(k_{i}\right)}(0)+\sum_{j=0}^{k_{i}-1} \alpha_{i j} f^{(j)}(0) & i=1,2, \cdots, \mu \\
k_{1}>k_{2}>\cdots>k_{\mu} \\
B_{i}(f)=f^{\left(l_{i}\right)}(1)+\sum_{j=0}^{l_{i}-1} \alpha_{i j} f^{(j)}(1) & i=1,2, \cdots, \mu \\
l_{1}>l_{2}>\cdots>l_{\mu}
\end{array}
$$

then, if $S$ is any closed operator whose domain consists of $2 \mu-2$ times differentiable functions $f$ with $f^{(2 \mu-2)}(x) \in \mathscr{L}_{2}(0,1), T+S$ is a spectral operator and, if $E\left(\lambda_{k}\right)$ is its spectral measure, then

$$
I=\sum_{k=1}^{\infty} E\left(\lambda_{k}\right)
$$

In particular, one may make the following choice for $S$ :

$$
q_{2}(x) \frac{d^{2 \mu-2}}{d x^{2 \mu-2}}+q_{3}(x) \frac{d^{2 \mu-3}}{d x^{2 \mu-3}}+\cdots+q_{2 \mu}(x)
$$

where the coefficients $q_{i}(x) \in \mathscr{L}_{2}(0,1)^{2}$. More generally, $S$ may be chosen in the form:

$$
Q_{2} \frac{d^{2 \mu-2}}{d x^{2 \mu-2}}+Q_{3} \frac{d^{2 \mu-3}}{d x^{2 \mu-3}}+\cdots+Q_{2 \mu}
$$

2 Note that the theorem actually holds for the wider class of boundary value problems in which the $2 \mu-1$ th derivative can be eliminated by a standard change of dependent variable [7, p. 72], 
where $Q_{i}$ is any bounded operator in $\mathscr{L}_{2}(0,1)$.

Application of Theorem 2 shows that if $f \in \mathscr{L}_{2}(0,1)$ and $E\left(\lambda_{k}\right)$ are the idempotents corresponding to $T+S$, then the series expansion

$$
f=\sum_{k=1}^{\infty} E\left(\lambda_{k}\right) f
$$

converges in $\mathscr{L}_{2}(0,1)$ norm.

An additional consequence of Theorem 3 and Corollary 2 of $[9, p$. $448]$ is the following.

Corollary 1. If $f \in C^{2 \mu-1}, f^{(2 \mu-1)}(x)$ is absolutely continuous, $f^{(2 \mu-1)}(x)$ $\in \mathscr{L}_{2}(0,1)$, and $f(x)$ satisfies the boundary conditions above, then $f$ can be expanded in the series

$$
f=\sum_{k=1}^{\infty} E\left(\lambda_{k}\right) f
$$

where convergence is in the sense that, letting

$$
S_{n}(x)=\sum_{k=1}^{n} E\left(\lambda_{k}\right) f
$$

we have

$$
\begin{aligned}
\lim _{n \rightarrow \infty} & \left\{\int_{0}^{1}\left|f(x)^{(2 \mu)}-S_{n}^{(2 \mu)}(x)\right|^{2} d x\right\}^{1 / 2} \\
& +\max _{0 \leqq x \leqq 1} \max _{0 \leqq i \leqq 2 \mu-1}\left|f^{(i)}(x)-S_{n}^{(i)}(x)\right|=0 .
\end{aligned}
$$

\section{REFERENCES}

1. S. Banach, Theorie des operations lineaires. Warszawa 1932.

2. G. D. Birkhoff, Boundary value and expansion problems of ordinary linear differen tial equations. Trans. Amer. Math. Soc. 9 (1908), 373-395.

3. N. Dunford, Spectral theory II. Resolutions of the identity. Pacific J. Math. 2 (1952), 559-614.

4. - Proceedings of Symposium on Spectral Theory and Differential Protlems. Stillwater, Oklahoma, 1951.

5. ___ Spectral Operators. Pacific J. Math. 4 (1954), 321-361.

6. E. Hille, Functional analysis and semi-groups. Amer. Math. Colloquium Publications, 31 (1948).

7. E. Kamke, Differentialgleichungen. Leipzig 1492.

8. E. R. Lorch, Bicontinuous linear transformations in certain vector spaces. Bull. Amer. Math. Soc. 45 (1939), 564-569.

9. J. Schwartz, Perturbations of spectral operators and applications. I. Bounded perturbations. Pacific J. Math. 4 (1954), 415-458. 
10. Béla v. Sz.-Nagy, Spektraldarstellung linearer Transformationen des Hilbertschen Raumes. Ergebnisse der Mathematik und ihrer Grenzgebiete 5, Berlin 19421.

11. J. Tamarkin, Sur quelques points de la théorie des équations differentielles linéaires ordinaires et sur la généralisation de la série de Fourier. Rend. Circ. Mat. Palermo 34 (1912), 345-382.

12. F. Wolf, Analytic perturbation of operators in Banach spaces. Math. Ann. 124 (1952), 317-333.

Bell Telephone laboratories, Murray hill, New Jersey 



\title{
PACIFIC JOURNAL OF MATHEMATICS
}

\author{
EDITORS
}

H. L. Royden

Stanford University

Stanford, California

R. A. Beaumont

University of Washington

Seattle 5, Washington
A. L. Whiteman

University of Southern California

Los Angeles 7, California

E. G. Straus

Unıversity of California

Los Angeles 24, California

\section{ASSOCIATE EDITORS}

\author{
E. F. BECKENBACH \\ C. E. BURGESS \\ M. HALL \\ E. HEWITT
}

\author{
A. HORN \\ V. GANAPATHY IYER \\ R. D. JAMES \\ M. S. KNEBELMAN
}

L. NACHBIN
I. NIVEN
T. G. OSTROM
M. M. SCHIFFER

G. SZEKERES

F. WOLF

K. YOSIDA

\section{SUPPORTING INSTITUTIONS}

UNIVERSITY OF BRITISH COLUMBIA

CALIFORNIA INSTITUTE OF TECHNOLOGY

UNIVERSITY OF CALIFORNIA

MON'TANA STATE UNIVERSITY

UNIVERSITY OF NEVADA

OREGON STATE COLLEGE

UNIVERSITY OF OREGON

UNIVERSITY OF SOUTHERN CALIFORNIA

\author{
STANFORD UNIVERSITY \\ UNIVERSITY OF UTAH \\ WASHINGTON STATE COLLEGE \\ UNIVERSITY OF WASHINGTON \\ AMERICAN MATHEMATICAL SOCIETY \\ CALIFORNIA RESEARCH CORPORATION \\ HUGHES AIRCRAFT COMPANY \\ THE RAMO-WOOLDRIDGE CORPORATION
}

Mathematical papers intended for publication in the Pacific Journal of Mathematics should be typewritten (double spaced), and the author should keep a complete copy. Manuscripts may be sent to any of the editors. All other communications to the editors should be addressed to the managing editor, E. G. Straus at the University of California, Los Angeles 24, California.

50 reprints per author of each article are furnished free of charge; additional copies may be obtained at cost in multiples of 50 .

The Pacific Journal of Mathematics is published quarterly, in March, June, September, and December. The price per volume (4 numbers) is $\$ 12.00$; single issues, $\$ 3.50$. Back numbers are available. Special price to individual faculty members of supporting institutions and to individual members of the American Mathematical Society: $\$ 4.00$ per volume; single issues, $\$ 1.25$.

Subscriptions, orders for back numbers, and changes of address should be sent to Pacific Journal of Mathematics, 2120 Oxford Street, Berkeley 4, California.

Printed at Kokusai Bunken Insatsusha (International Academic Printing Co., Ltd.), No. 10, 1-chome, Fujimi-cho, Chiyoda-ku, Tokyo, Japan.

PUBLISHED BY PACIFIC JOURNAL OF MATHEMATICS, A NON-PROFIT CORPORATION

The Supporting Institutions listed above contribute to the cost of publication of this Journal, but they are not owners or publishers and have no responsibility for its content or policies. 


\section{Pacific Journal of Mathematics}

\section{Vol. 7, No. $3 \quad$ March, 1957}

Silvio Aurora, Multiplicative norms for metric rings............... 1279

Ross A. Beaumont and John Richard Byrne, On the construction of

$R$-modules and rings with polynomial multiplication ............ 1305

Fred Brafman, An ultraspherical generating function . . . . . . . . . . . 1319

Howard Ernest Campbell, On the Casimir operator ............... 1325

Robert E. Edwards, Representation theorems for certain functional

operators..................................... 1333

Tomlinson Fort, The five-point difference equation with periodic

coefficients ..................................... 1341

Isidore Heller, On linear systems with integral valued solutions......... 1351

Harry Hochstadt, Addition theorems for solutions of the wave equation in

parabolic coordinates ................................ 1365

James A. Hummel, The coefficient regions of starlike functions . . . . . . . . 1381

Fulton Koehler, Estimates for the eigenvalues of infinite matrices ......... 1391

Henry Paul Kramer, Perturbation of differential operators ............. 1405

R. Sherman Lehman, Development of the mapping function at an analytic

corner .......................................... 1437

Harold Willis Milnes, Convexity of Orlicz spaces.................. 1451

Vikramaditya Singh, Interior variations and some extremal problems for certain classes of univalent functions . . . . . . . . . . . . . . . . 1485

William Lee Stamey, On generalized euclidean and non-euclidean spaces ............................................. 1505

Alexander Doniphan Wallace, Retractions in semigroups .............. 1513

R. L. Wilder, Monotone mappings of manifolds .................. 1519 